\section{SAT0040 RHEUMATIC DISEASES IN PATIENTS WITH VITILIGO: A STUDY ON 28 CASES}

${ }^{1}$ I Olivieri, ${ }^{1} \mathrm{G}$ Ciancio, ${ }^{1} \mathrm{~A}$ Padula, ${ }^{1} \mathrm{~L}$ La Civita, ${ }^{2} \mathrm{~A}$ Piccirillo, ${ }^{2} \mathrm{~F}$ Ricciuti, ${ }^{3} \mathrm{~F}$ Cantini, ${ }^{3} \mathrm{~L}$ Niccoli, ${ }^{4} \mathrm{C}$ Salvarani. ${ }^{1}$ Rheumatology Department of Lucania; ${ }^{2}$ Dermatology Unit, San Carlo Hospital, Potenza; ${ }^{3}$ Rheumatic Disease Unit, Prato Hospital, Prato; ${ }^{4}$ Rheumatic Disease, Arcispedale S. Maria Nuova, Reggio Emilia, Italy

\subsection{6/annrheumdis-2001.392}

Background Recently, we have demonstrated in a case-control study that vitiligo and spondyloarthropathy (SpA) do not coexist by chance in the same patient. ${ }^{1}$ Of the 234 patients with SpA seen in the study period 8 (3.4\%) were found to be suffering from type A vitiligo. On the contrary, among the 468 control patients only $5(1.06 \%)$ had type A vitiligo. The difference was statistically significant $(\mathrm{p}<0.05)$.

Objectives To establish the reasons for the rheumatological consultation in consecutive patients with vitiligo seen for the first time in our department.

Methods All patients that showed visible type A vitiligo in periorificial sites (around mouth, nose, eyes) and/or over the extensor surfaces of hands and wrists, seen for the first time in our department from January 1999 to 31 December 2000, were evaluated clinically. X-ray and laboratory examinations were performed when necessary.

Results Twenty-eight patients with visible vitiligo were seen. Of these, 4 had inflammatory rheumatic diseases associated with vitiligo (2 elderly onset undifferentiated SpA, 2 psoriatic arthritis, 1 rheumatoid arthritis together with lupus profundus). Five patients had inflammatory rheumatic diseases with dubious relationships with vitiligo ( 3 polymyalgia-like syndrome, 1 seronegative chronic polyarthritis, 1 severe peripheral enthesitis). The remaining 17 patients had vitiligo unrelated rheumatic diseases including osteoarthritis, mechanical low back pain, herniated disc, spondylolisthesis, condrocalcinosis, rotator cuff tendinitis, olecranic bursitis and trigger finger. Seven out of the 28 patients had diseases of the thyroid gland and 1 alopecia areata.

Conclusion The results of the present study confirm the association between vitiligo and SpA. Some patients with vitiligo have undifferentiated inflammatory rheumatic diseases, which need to be followed up.

\section{REFERENCE}

1 Padula A, et al. Association between vitiligo and spondyloarthropathy. I Rheumatol. 2001;28, in press

\section{SAT0041 VALIDITY OF THE APPLICATION OF A DIAGNOSTIC PROTOCOL ACCORDING TO PATTERNS OF CLINICAL PRESENTATION OF UVEITIS TO ESTABLISH DIAGNOSIS}

${ }^{1} \mathrm{G}$ Bonilla Hernán, 'S Muñoz Fernandez, ${ }^{2} \mathrm{~V}$ Hidalgo, ${ }^{1} \mathrm{~J}$ Fernandez Melón, ${ }^{2} \mathrm{~A}$ Schlincker, ${ }^{2} \mathrm{~A}$ Fonseca, ${ }^{1} \mathrm{~F}$ Gamero Ruiz, ${ }^{2} \mathrm{~B}$ Damas, ${ }^{1} \mathrm{E}$ Martín Mola. ${ }^{1}$ Rheumatology; ${ }^{2}$ Oftalmology, Hospital La Paz, Madrid, Spain

10.1136/annrheumdis-2001.393

\section{Background}

Objectives To validate the efficiency of the application of a diagnostic protocol based on the patterns of presentation of uveitis to establish the diagnosis.

Methods Our Uveitis Clinic is composed of a multidisciplinary team of Ophatalmologists and Rheumatologist.

We defined a clinical diagnostic protocol for the uveitis, based on the frequency of syndromes found in the literature in each clinical pattern of uveitis and the symptoms of the patients. We routinarelly performed a careful history, a complete ophtalmologic examination, hemogram, biochemistry, ESR, a fluorescent treponemal antibody absorption test, an urinary test and a chest $\mathrm{X}$ Ray. Other diagnostic procedures were ordered according to a established protocol based on the clinic patterns of presentation of uveitis. Data from the patients were prospectively recorded from June of 1997 to October 2000.

Results 366 patients were included, 40 of them (10.7\%) were lost of follow-up,146 of the 336 who completed the study were diagnosed of idiopahatic uveitis (43.4\%),70 (21\%) had some type of spondyloarthopaty (30 patients were diagnosed in our uveitis clinic), 33 (9.8\%) were secondary to infections (23 herpes, 6 toxoplasmosis, 3 tuberculosis, 1 candida), 3 were masquerade syndromes (1 lung cancer and 2 linphoproliferative diseases), $6(1.7 \%)$ were traumatic, $47(14 \%)$ were ophtalmologic syndromes, $21(6.25 \%)$ uveitis were secondary to other rheumatic diseases (8 Behçet diseases, 8 Sarcoidosis,4 Sjögren's syndrome and one juvenile chronic arthritis) and 2 were TINU syndromes.

Conclusion We have achieved the etyology of the uveitis in $56.6 \%$. A greater percentage than those found in other series in which the diagnostic procedures have not been prestablished. In addition, it could have a better cost-effective impact and avoid the number of consultations between different specialists. We believe that this is the first study in which a clinical protocol of diagnostic procedures has been validated in patients with uveitis.

\section{SAT0042 CLINICAL FEATURES OF PSORIATIC ARTHRITIS WITH A DELAYED ONSET}

MD Lopez-Montilla, A Escudero, M Romero, V Perez, C Castro, J Gonzalez, MC MuñozVillanueva, E Collantes. Rheumatology Service, Hospital Universitario Reina Sofia, Cordoba, Spain

10.1136/annrheumdis-2001.394

Background The influence of patient age at onset on the clinical expression of rheumatic diseases has been widely demonstrated in relation to pathologies such as rheumatoid arthritis and ankylosing spondylitis. Such influence, however, has only occasionally been considered in connexion with psoriatic arthritis (PsoA).

Objectives To identify potential differences among the clinical manifestations of PsoA depending on patient age at the onset of the disease.

Methods Ninety-six consecutive patients diagnosed with PsoA as per the criteria of Moll and Wright were split into two groups according to whether their early symptoms of the disease appeared before or after the age of 60 . The clinical features of both groups were assessed.

Results The following Table 1 shows the differential clinical features of the two patient groups, established according to onset age.

\begin{tabular}{lll} 
Abstract SAT0042 Table 1 & \\
\hline & $\begin{array}{l}\text { Group I } \\
\text { (under 60 years) }\end{array}$ & $\begin{array}{l}\text { Group II } \\
\text { (over 60 years) }\end{array}$ \\
\hline Number of patients & 84 & 12 \\
Gender & $48 \% \mathrm{M} / 52 \% \mathrm{~F}$ & $75 \% \mathrm{M} / 25 \% \mathrm{~F}$ \\
Family history & $35 \%$ & $17 \%$ (paternal) \\
Currently prevailing form & $43 \% \mathrm{SpA} / 34.5 \% \mathrm{PA}$ & $17 \% \mathrm{SpA} / 45 \% \mathrm{PA}$ \\
Dactylitis & $50 \%$ & $25 \%$ \\
Sacroilitis & $41.5 \%$ & $17 \%$ (unsymmetric)
\end{tabular}




\begin{tabular}{|c|c|c|}
\hline HLA-B27 positive & $7.7 \%$ & none \\
\hline
\end{tabular}

Conclusion PsoA patients with a delayed onset of the disease exhibit clinical symptoms that differ clearly from those of the other PsoA patients.

\section{SAT0043 ANKYLOSING SPONDYLITIS AND CARDIAC DISEASES}

${ }^{1} \mathrm{FM}$ Santos, ${ }^{2} \mathrm{~A}$ Alves, ${ }^{3} \mathrm{C}$ Catarino, ${ }^{3} \mathrm{~L}$ Bento, ${ }^{3} \mathrm{C}$ Cotrim, ${ }^{3} \mathrm{M}$ Carrageta, ${ }^{1} \mathrm{JC}$ Silva. ${ }^{1}$ Rheumatology; ${ }^{2}$ Internal Medicine; ${ }^{3}$ Cardiology, Garcia de Orta Hospital, Almada, Portugal

10.1136/annrheumdis-2001.395

Background Ankylosing Spondylitis (AS) and other spondyloarthropathies (SPA), are associated with certain cardiologic problems.

Objectives To identify coexisting AS and SPA in selected cardiac patients (pts) and to investigate the cardiac status of pts with an established diagnosis of AS.

Methods An observational study involving two pt. groups was undertaken: Group 1 were young and middle-aged patients ( $<50$ years at time of diagnosis), followed in the cardiology department for aortic valve disease and/or with a permanent pacemaker implanted. All pts were interviewed and examined. A pelvic X-ray and blood analysis for HLA B27 was performed. Group 2 were pts with an established diagnosis of AS and no clinical evidence of cardiovascular disease. A full history, clinical examination, 24-h Holter and doppler echocardiography were performed. Cardiac abnormalities were related to clinical features of AS.

Results Group 1: 14 pts with either an aortic valve replacement (AVR), aortic valve pathology and/or a permanent pacemaker were studied. 12 men and 2 women, with a mean age of 40 years (range 22-53) were observed. 9 pts had an AVR, 2 had aortic valva (AV) stenosis and 5 had a permanent pacemaker implamted. 2 of the pts had both an AVR and a permanent pacemaker. 1 of these pts was definitely diagnosed with AS and 2 pts were diagnosed as undifferentiated SPA. Group 2: 9 men and 8 women, with an average age of 43 years (min 23 and max 61) were included. Disease duration varied from 1 to 20 years with a mean of 8 . Four (24\%) pts had echocardiographic evidence of aortic valve thickening and regurgitation. Two pts also had left atrial dilatation. Holter exams were performed in 15 of the 17 pts. No significant conduction abnormalities or arrhythmias were apparent. The 4 pts with echocardiographic changes were older ( $>50$ years in 3 cases) or had a high Basmi index.

Conclusion Young pts with AV disease or conduction disorders should be screened for AS. Regular cardiac monitoring of AS pts may help prevent the long term cardiac complications associated with this condition.

\section{SAT0044 CLASS II MHC MOLECULES IN PATIENTS WITH SPORADIC ANKYLOSING SPONDYLITIS IN THE SOUTHERN EUROPEAN POPULATION}

VC Perez-Guijo, E Muñoz-Gomariz, A Escudero, M Romero, MA Aguirre, J Gonzalez, FG Martinez, MC Muñoz-Villanueva, C Castro, R Solana, J Peña, E Collantes. Rheumatology Service, Hospital Universitario Reina Sofia, Cordoba, Spain
Background To evaluate the relationship between the presence of HLA-DRB1 genes and predisposition to AS in a demographically well-defined population.

Objectives 113 individuals with sporadic (non-familial) Ankylosing Spondylitis (AS) from six different cities and 747 bone marrow donors (BMD) as control group.

Methods All individuals were typed for HLA-B27 by either flow cytometry with monoclonal antibodies or PCR-SSP, as well as for HLA-DRB using the Dynal ELITM SSO HLA-DRB Test (Dynal AS, Oslo, Norway). The Inno-Lipa DRB key and DRB Decoder (Innogenetics NV Zwijndrecht, Belgium), which is based on PCR-SSO technology, was used for high-resolution HLA -DRB typing.

Results Table 1 shows the results.

\begin{tabular}{|c|c|c|c|c|c|c|}
\hline & $\begin{array}{l}\text { B27(+) } \\
\text { AS (n } \\
=94)\end{array}$ & $\begin{array}{l}\text { B27(+) } \\
\text { BMD (n } \\
=56)\end{array}$ & $\begin{array}{l}\mathrm{p}^{*} \text { and } \mathrm{OR} \\
(95 \% \mathrm{Cl})\end{array}$ & $\begin{array}{l}\text { B27(-) } \\
\text { AS (n } \\
=19)\end{array}$ & $\begin{array}{l}\text { B27(-) } \\
\text { BMD (n = } \\
691)\end{array}$ & $\begin{array}{l}\mathrm{p}^{*} \text { and } \mathrm{OR} \\
(95 \% \mathrm{Cl})\end{array}$ \\
\hline DRB1*01 & $\begin{array}{l}32 \\
(34 \%)\end{array}$ & $\begin{array}{l}31 \\
(55.3 \%)\end{array}$ & $\begin{array}{l}0.022 \text { and } 0.42 \\
(0.2-0.8)\end{array}$ & $8(42 \%)$ & $\begin{array}{l}140 \\
(20 \%)\end{array}$ & $\begin{array}{l}0.042 \text { and } 2.9 \\
(1-8)\end{array}$ \\
\hline DRB1 *04 & $\begin{array}{l}12 \\
(13 \%)\end{array}$ & $15(27 \%)$ & ns and 0.4 & $5(26 \%)$ & $\begin{array}{l}169 \\
(24 \%)\end{array}$ & ns and 1.1 \\
\hline DRB1*06 & $\begin{array}{l}23 \\
(24 \%)\end{array}$ & $7(12 \%)$ & ns and 2.3 & $4(21 \%)$ & $\begin{array}{l}218 \\
(31 \%)\end{array}$ & ns and 0.58 \\
\hline DRB1 *09 & $3(3 \%)$ & $3(5 \%)$ & ns and 0.58 & $2(10 \%)$ & $15(2 \%)$ & ns and 5.3 \\
\hline
\end{tabular}

Conclusion The incidence of the DRB $1 * 01$ antigen in the studied population is significantly higher in B27(+) healthy individuals (BMD) than in $\mathrm{B} 27(+)$ AS patients. Also the incidence of this antigen is significantly higher in B27(-) AS patients than B27 (-) healthy individuals. This could implicate that in the absence of B27, the DRB1*01 antigen could take part in the development of the disease.

\section{SAT0045 SPONDYLODISCITIS IN ANKYLOSING SPONDYLITIS}

${ }^{1} \mathrm{P}$ Borman, ${ }^{1} \mathrm{U}$ Seckin, ${ }^{1} \mathrm{H}$ Gunduz, ${ }^{2} \mathrm{~N}$ Barca, ${ }^{2} \mathrm{~L}$ Damgaci. ${ }^{1}$ Department of Physical Medicine and Rehabilitation; ${ }^{2}$ Radiology, Numune Education and Research Hospital, Ankara, Turkey

\subsection{6/annrheumdis-2001.397}

Background Spondylodiscitis is a rare but well-known complication of ankylosing spondylitis (AS).

Objectives The aim of this study was to evaluate the incidence of spondylodiscitis and to determine the clinical and radiological appearance in a group of patients with AS.

Methods Thirty-two patients with a mean age of $36.4 \pm 11.4$ years were recruited to the study. Demographical, clinical and radiological characteristics of the patients were recorded. Bone scintigraphic evaluation and computerised tomography (CT) of the suspected lesions were determined.

Results Five (15.6\%) patients had radiographic abnormalities including irregular narrowed disc space borders and sclerosis of the adjent vertebral bodies in the thoracic and lumbar regions. All these patients had low back pain the clinical activity parameters were higher in $3(9.3 \%)$ of 5 patients. No fracture or pseudoarthrosis were observed. Technetium scan showed increased uptake in the vertebral disc space and vertebral end plates (L4- 